\title{
COUP D'CEIL SUR L'ÉCOLE FRANÇAISE DE GÉOGRAPHIE AU MILIEU DU XX'e SIÈCLE
}

\author{
Bernard Grandjean
}

Une année après la disparition d'Emmanuel de Martonne (1873-1955) qui «restera dans le souvenir de tous les Géographes français, un des plus grands serviteurs de la Géographie française» (16) ${ }^{1}$, l'Information géographique, sous la direction de MM. G. Снавот, Professeur à la Sorbonne, R. Clozier, Inspecteur général de l'Education Nationale, et de $M^{\mathrm{me}} \mathrm{J}$. Beaujeu-Garnier, Professeur à la faculté des Lettres de Lille, assurés du concours d'une quarantaine de spécialistes, publie un magistral tableau de la Géographie française (1).

Ce bilan, qui n'ignore rien des tendances nouvelles, entend «rester fidèle à l'unité de la géographie qui, en trop s'éparpillant, perdrait jusqu'à sa raison d'être»2. Il est surtout, aujourd'hui, le digne hommage rendu à un demi-siècle de recherche, de travail et d'effort au service de la science d'abord, d'une meilleure - et par conséquent d'une plus juste et plus vraie - connaissance des faits naturels et des diverses régions de la Terre ensuite, des hommes et des peuples enfin. Demain, ce «rapport sur la géographie française» 3 constituera une base de départ des plus solides pour le jeune chercheur, le point de repère de tous ceux qui œuvrent «entraînés par un courant d'idées nouvelles»4, tant il est vrai qu'en face du malaise que peut créer une spécialisation de plus en plus indispensable et tyrannique, «un regard en arrière sera toujours le remède le plus sûr ». (4o).

Les directeurs de la revue l'Information géographique et leurs collaborateurs ont désiré «faire le point» de l'Ecole française de géographie. Non seulement ils y sont pleinement parvenus, mais encore, ils ont du même coup créé un centre de ralliement où viendront se rencontrer, se confronter et se définir les opinions et les connaissances les plus diverses, un centre de ralliement d'où pourront repartir enrichis et fortifiés, les géographes de toutes les nations avides d'une description et d'une tentative d'explication toujours renouvelées de la Terre et de l'Homme.

Il est impossible de résumer systématiquement les quarante-deux contributions rassemblées sous le titre de La Géographie française au milieu du $X X^{e}$ siècle (1), car les disciplines sont trop diverses, les faits présentés trop riches, les auteurs invoqués trop nombreux. Nous nous bornerons à regrouper les sciences géographiques, à signaler quelques œuvres capitales et à souligner les grandes tendances (5) qui se sont imposées ou qui se dessinent.

La géographie en général et la géographie française en particulier n'ont pas toujours connu l'épanouissement qui les caractérise aujourd'hui ${ }^{6}$, ni surtout, poursuivi toujours les mêmes recherches et les mêmes buts qu'en ce milieu du $X X^{\mathrm{e}}$ siècle ${ }^{\top}$.

La géographie moderne, telle que l'a définie de façon si magistrale E. DE MARTONNE en $1909^{8}$ est l'aboutissement d'une longue évolution dont on retrouve des traces dès la Première Ecole d'Alexandrie au III ${ }^{\mathbf{e}}$ siècle av. J.-C., évolution qui s'accen-

1 Les chiffres entre parenthèses () renvoient à la bibliographie publiée en pages $5+$ et 55 .

$2(1)$, p. 6.

3 La Géographie française au milieu du $\mathrm{XX}^{\mathrm{e}}$ siècle (1) a été publiée à l'intention du XVIII ${ }^{e}$ Congrès international de Géographie tenu à Rio de Janeiro au mois d'août 1956. Un premier rapport sur la Géographie française avait été présenté par E. DE MARTONNE en 1915, au Congrès de San Francisco. Ce rapport a été réédité en 1933.

4 (1), p. 5.

5 «On aurait tort de penser que la géographie est fixée une fois pour toutes dans sa structure et ses tendances; elle n'a que cinquante ans d'existence». A. Cholley (1), p. 25.

6 «Il est certain que la géographie française est actuellement dans une belle effervescence qui peut bien ressembler à de la prospérité ». M. Le Lannou. 1 art. in Le Monde, 10. 11. 56.

7 Dans l'Antiquité, géographie et cartographie furent souvent synonymes; essentiellement descriptive jusqu'à la fin du XIX ${ }^{\mathbf{e}}$ siècle, notre discipline ne reste trop souvent encore pour le grand public qu'une indigeste nomenclature, comme l'écrivait déjà L. Vivien dE SAINT-Martin en 1873.

8 «La géographie moderne envisage la répartition à la surface du globe des phénomènes physiques, biologiques et humains, les causes de cette répartition et les rapports locaux de ces phénomènes». E. DE Martonne (4o), p. 24. 
tuera au siècle dernier grâce au mérite des géographes allemands ${ }^{9}$. L'Ecole géographique française «est le résultat normal d'une période d'organisation qui a débuté avec $\mathrm{P}$. VIDAL DE LA Blache (1843-1918) vers la fin du XIX siècle et qui s'est prolongée pendant les vingt-cinq années suivantes grâce à l'action de ses élèves directs. Jusque-là dispersées, les principales branches de la géographie ont été regroupées en un même corps de discipline dont les géographes par leurs travaux ont souligné l'unité de méthode». De plus, ce regroupement a été effectué dans le cadre universitaire des facultés des lettres, ce qui n'est nullement une difficulté comme le montre pertinement $\mathrm{A}$. Cholley, directeur de l'Institut de Géographie de l'Université de Paris ${ }^{10}$.

L'unité de notre discipline ${ }^{11}$, créant du même coup l'unité de méthode, a favorisé son autonomie et par suite, une tendance au travail en profondeur qui répond d'ailleurs à un besoin accru de rigueur scientifique. Cette unité se traduit par un certain état d'esprit que caractérisent: «l'esprit de synthèse, le sens des ensembles localisés, celui des interdépendances, des solidarités à l'intérieur des complexes», esprit géographique qui à son tour pénètre bon nombre de disciplines voisines ${ }^{12}$.

Mais, unité ne veut pas dire uniformité. La vitalité des études de géographie régionale, où la géographie française «occupe peut-être dans ce domaine la première place dans le monde ${ }^{13}$, l'importance, le nombre et la diversité des travaux de géographie générale forment un autre trait caractéristique de la géographie française moderne. Si la géographie régionale s'est développée la première et contribue pour une large part à l'édification de la géographie générale, cette dernière envisage la réalité à l'échelle du globe entier. C'est elle également qui permet, grâce au dépouillement des éléments accidentels et locaux, l'explication de la réalité géographique régionale.

La géographie explicative impose de sérieuses obligations, notamment un contact continuel avec les disciplines parallèles: physique du globe, sciences naturelles, sciences humaines. Si le contact avec l'histoire est aisé ${ }^{14}$, s'il est nécessaire du point de vue de l'évolution, en particulier en géographie humaine, il a perdu cette primauté qui bien souvent faisait de la géographie un simple auxiliaire de la discipline de Clio! Le contact s'est élargi avec les sciences proprement dites: géologie, minéralogie, biologie. Il s'agit non seulement pour le géographe du $X X^{\mathrm{e}}$ siècle d'une véritable «formation complémentaire régulière», mais encore d'établir des relations de plus en plus marquées avec les autres jeunes chercheurs: météorologistes, hydrologues, etc., pour le plus grand profit des deux catégories de disciplines ${ }^{15}$.

Unité, autonomie, travail en profondeur et en collaboration tant dans le domaine de la géographie régionale que dans celui de la géographie générale témoignent d'une intense évolution interne de la géographie. Or, depuis les années quarante, une «série de mutations ont changé les conditions de nos recherches. Les progrès de la technique nous font un monde nouveau», et, de nouvelles techniques engendrent de nouvelles

9 L'Ecole française n'oublie pas le mérite des maîtres allemands. Les pages consacrées à A. Humboldt et à C. Ritter en 1909 par E. De Martonne, en 1956 par A. Meynier en sont la meilleure preuve.

10 voir A. Cholley (1) p. 14.

${ }^{11}$ E. DE Martonne, en particulier, a toujours proclamé cette unité: « Nous croyons toujours à l'unité de la Science géographique... (40), Préface de la 4e édition, 1925; "La géographie physique est le fondement nécessaire de toute géographie. (40), Préface de la 6e édition, 1940.

12 voir M. SORRE (1) p. 9 et 10.

13 voir D. FAUChER (1) p. 302.

14 Pour diverses raisons: les maitres de l'Ecole française étaient de formation historique; histoire et géographie se rattachent à la même faculté, etc.

15 «L'avenir semble être aux recherches en équipes, où chacun apporterait le fruit de son effort particulier et profiterait de l'apport des autres pour élaborer sa propre synthèse.» E. JuILLARD (1) p. 166. C'est dans l'immense domaine en devenir de la géographie appliquée que le travail en collaboration, en équipes, aura plus particulièrement, à notre avis, la possibilité d'être fécond. 
recherches ${ }^{16}$. En même temps, les horizons de recherche s'élargissent, et, alors que hier le jeune Français se limitait volontiers à la France, à l'Afrique du Nord et aux Etats européens voisins, la bibliographie géographique française couvre aujourd'hui la planète, du Grœnland aux terres équatoriales, de l'Amérique du Sud à l'Afrique et à l'Asie, en passant par le Proche-Orient ${ }^{17}$.

Animée par «un véritable bouillonnement d'idées» ${ }^{18}$, l'Ecole française de géographie, repoussant avec la même énergie «toute spécialisation excessive» et tout dogmatisme, ouverte aux techniques nouvelles, consciente des difficultés de concilier entre autres les exigences de la synthèse régionale avec la rigueur de l'analyse scientifique, reste fidèle à l'esprit de ses premiers maîtres et à sa «vocation d'humanisme» que lui impose sa mission de décrire rationnellement la Terre.

Depuis la parution en 1909 du Traité d'E. De Martonne (40), la Géographie Physique a connu, tant par l'enseignement que par les travaux de feu l'illustre directeur de l'Institut de géographie de Paris, un développement extraordinaire comme en témoignent les douze communications réunies sous la couverture de l'Information géographique.

Dans la première, H. BAULIG ${ }^{19}$ s'attache à fixer les origines de la géomorphologie en France, œuvre souvent de géologues (E. DE Beaumont, A. DE Lapparent, E. DE MARGERIE), de topographes (G. DE LA NOË), puis son développement marqué par les travaux d'une quinzaine de chercheurs - dont bon nombre aujourd'hui, professeurs d'Université - sous la conduite d'E. DE Martonne.

Au cours des vingt dernières années, la géomorphologie française a subi une évolution assez remarquable ${ }^{20}$. Dépassant le stade de la description et de l'analyse des formes du relief, A. Cholley, P. Birot, A. Cailleux, J. Tricart, J. BeaujeuGarnier, A. Guilcher et d'autres ont introduit le travail en laboratoire, préoccupés surtout des «processus et des vicissitudes de l'évolution globale du relief», préoccupation qui a mis en valeur d'abord, l'importance du facteur climat, ensuite, la notion du dynamisme intégral de la morphologie.

Le fractionnement en disciplines séparées se révèle comme une autre tendance de l'étude de la géomorphologie en France au cours de ce dernier demi-siècle, comme en font foi les titres des cinq articles suivants: la morphologie désertique 21 , la géomorphologie glaciaire et périglaciaire ${ }^{22}$, la morphologie volcanique ${ }^{23}$, le relief karstique ${ }^{24}$ et la morphologie littorale 25 .

Si la morphologie est toujours en tête des recherches, la climatologie française $\mathbf{2 6}$, après avoir connu une éclipse vers 1930, renaît sous l'influence de travaux étrangers - scandinaves et germaniques - d'une part, des nouvelles perspectives de la morphologie d'autre part. Mais, c'est surtout le secteur économique qui réclame de nouvelles

16 Que l'on songe seulement à l'extraordinaire moisson scientifique dans les régions polaires, ou aux nouveaux moyens de mesure auxquels ont recours la granulométrie ou l'étude de la désagrégation des roches, par exemple.

${ }_{17}$ C'est ici le lieu de mentionner l'intéressante collection de Textes et documents réunis par H. Lauga (33).

18 L'enseignement de la géographie à tous les degrés, et notamment l'enseignement supérieur bénéficie au premier chef de cet épanouissement. En 1920, 15 Universités françaises comptaient 23 professeurs, chargés de cours et assistants; en 1955, 71 !

${ }_{19}$ H. Baulig. La géomorphologie en France jusqu'en 1940. (1), pp. 27-35.

20 J. Beaujeu-Garnier. Les nouvelles tendances de la morphologie française. (1), pp. $37-42$.

21 R. CAPOT-REY. Recherches récentes et tendances nouvelles en morphologie désertique (bibliographie). (1), pp. $43-51$.

${ }_{22}$ J. TRICART. Géomorphologie glaciaire et périglaciaire. (1), pp. 53-58.

23 M. Derruau. Morphologie volcanique. (1), pp. 59-63.

24 A. Blanc. Le relief karstique. (1), pp. 65-69.

25 A. Guilcher. Morphologie littorale. (1), pp. 77-82.

26 C. P. PéguY. La climatologie française depuis trente ans. (1), pp. 83-85. 
connaissances climatiques, qu'il s'agisse de l'étude des précipitations dans le domaine de l'énergie hydro-électrique ou des micro-climats en agriculture, par exemple.

Grâce à M. PARdÉ, la potamologie est un des beaux fleurons de la géographie physique française. Le mémoire du professeur de Grenoble 27 est trop complet pour être résumé, notamment ses références bibliographiques. Cependant, c'est le lieu de signaler ici une fois encore l'importance de la collaboration entre géographes, hommes de sciences et ingénieurs d'abord, entre météorologie - précipitations - morphologie érosion et transport - mathématiques - emploi des méthodes statistiques - services publics - électricité, navigation - et hydrologie ensuite.

Tombée très bas à la fiǹ de la guerre 1914-1918, l'océanographie 28 connaît «un remarquable renouveau depuis quelques années». La création de nouveaux laboratoires ${ }^{29}$, les Expéditions polaires françaises, des initiatives privées comme celle du Cdt. Cousteau avec la Calypso, les Services publics enfin ${ }^{30}$ lui ont redonné vie. Océan Indien, Méditerranée, mers boréales, golfe de Gascogne, bassin du Cap Vert ont vu les chercheurs français se pencher sur leur hydrologie, leur bathymétrie, leur optique et leur hydrodynamique, sans oublier leur morphologie ${ }^{31}$, leur géologie et leur biologie sous-marines. Parmi les nombreux auteurs, bornons-nous à signaler le nom d'A. Guilcher (31), parmi les nombreuses publications, le Bulletin du Comité central d'Océanographie et d'Etude des Côtes, qui a diffusé un grand nombre de contributions et signalé les autres.

Cette rapide revue suffit à montrer que toutes les branches de la géographie physique retiennent l'attention des géographes français, sans oublier la biogéographie et la géographie botanique ${ }^{32}$. Il en est de même en géographie humaine.

L'homme, l'habitat, l'exploitation et la circulation ${ }^{33}$ restent au centre des études de Géographie Humaine au milieu du $X X^{\mathbf{e}}$ siècle. C'est la place que leur avaient assignée, dès la création de la nouvelle discipline, J. BRUnhes (11) et P. VIDAL DE La Blache ${ }^{34}$, plus tard A. Demangeon (20), aujourd'hui, M. Sorre (45), P. George (27-28), J. Beaujeu-Garnier (8), R. Capot-Rey (12), G. Chabot (14), P. Lavedan (34), et M. Le Lannou (37).

Dans La Géographie française au milieu de $X X^{\mathrm{e}}$ siècle, $\mathrm{M}^{\mathrm{me}}$ Beaujeu-Garnier traite Les développements de la géographie de la population ${ }^{35}$ en un article riche en références bibliographiques tandis que A. Perpillou signe une étude aussi brève que dense sur les Problèmes de l'habitat rural ${ }^{36}$. Le professeur à la Sorbonne rend hommage à A. Demangeon, rappelle ensuite le programme de recherches que ce dernier avait défini, démontre enfin que l'étude de l'habitat rural est loin d'être terminée ${ }^{37}$, l'influence des milieux physique et social étant variable dans le temps et selon les lieux, l'influence du maître du sol se révélant souvent plus importante que d'aucuns n'ont su ou voulu le voir.

27 M. Pardé. Les études d'hydrologie fluviale depuis un quart de siècle. (1), pp. 103-119.

28 A. Guilcher. Océanographie. (1), pp. 121-126.

${ }^{29}$ Création des laboratoires du Centre de recherches et d'études océanographiques (C.R.E.O.) à Antibes et à La Rochelle.

${ }^{30} \mathrm{La}$ Section des marées au Service hydrographique, par exemple.

31 voir ci-dessus note 25 , p. 47.

32 P. Birot. La géographie botanique en France dans les vingt dernières années. (1), pp. 99-102.

33 R. Clozier. La géographie de la circulation. (1), pp. 175-181.

${ }^{34}$ Le nom de Géographie humaine recouvre une des questions qui ont le plus anciennement préoccupé l'esprit humain : celle des rapports réciproques de la Terre et de l'Homme. P. VIDAL DE LA Blache (48).

35 (1), pp. $127-132$.

80 (1), pp. $133-141$.

37 “.. il est significatif qu'aucune étude synthétique n’ait encore été tentée... A. PERPII.L.OU (1), p. 133 . 
Discipline autrefois mineure, la géographie urbaine 38 connaît depuis une dizaine d'années un véritable renouveau. Si la tradition des monographies urbaines s'est poursuivie par de nombreuses publications sur Aurillac (1946), Sao Paulo (1953), Los Angelès (1953), Ganges (1953), Le Mans (1953), Toulouse (1954), «de nouvelles préoccupations sont apparues. On a cherché à systématiser une géographie urbaine, à fixer un champ d'action et des méthodes, ce qui n'avait jamais été fait, en France, tout au moins »39. Non seulement le géographe s'efforce par la comparaison de dégager des traits communs, mais encore, son enquête s'élargit, attirée et enrichie par les faits sociaux et démographiques, propulsée par l'extraordinaire extension urbaine de ces vingt dernières années. Mais la ville s'étend du côté de la campagne; l'étude de l'influence réciproque des régions rurale et urbaine s'impose à notre époque.

La géographie économique englobe un domaine si vaste, si général, qu'au cours de ce demi-siècle, elle s'est divisée en géographie agricole ${ }^{40}$, en géographie industrielle ${ }^{41}$ et même en géographie de l'énergie (26). Les études ainsi groupées nous apportent des pages montrant une documentation de plus en plus abondante, la capacité de l'action humaine de modifier les aptitudes naturelles, des techniques de recherches de plus en plus développées - pas toujours maîtrisées! 42 - des publications de plus en plus nombreuses sur des industries déterminées, les Industries de la la soie en France (18), par exemple, ou au contraire des études d'ensemble comme la Géographie des Textiles (2). Signalons encore le désir de plus en plus vif de voir les travaux de géographie agraire et industrielle «servir non seulement au progrès de nos connaissances, mais encore, au choix d'une politique d'aménagement du territoire».

Pour terminer ces quelques notes intéressant la géographie humaine, il faudrait encore avoir la possibilité de parcourir ces chemins toujours nouveaux - encore que très anciens - chemins qui conduisent à une meilleure connaissance de l'homme «en tant qu'être pensant», chemins de la géographie politique et de la géographie religieuse ${ }^{43}$ convergeant en une géographie sociale. Ces complexes difficiles à saisir mais qui sont les réalités auxquelles se heurtent les sociétés humaines ont été étudiés par des esprits aussi pénétrants que J. Ancel (3-4), R. Dion (22), J. Gottmann (30), A. SiEgFried (42-44), «le grand initiateur des études de géographie électorale», et qui a su si bien montrer l'importance du cadre géographique à côté de l'action des faits sociaux et religieux - P. Deffontaines (19), auteur d'un «véritable bilan des influences religieuses dans le domaine géographique».

Il faudrait enfin pouvoir présenter, mûrir ces trois pages particulièrement denses consacrées à une définition de la géographie historique 44 dont l'objet est d'expliquer les choses (géographiques) en retraçant leur genèse, seule possibilité pour l'homme moderne d'essayer de comprendre son présent et d'effleurer le voile qui couvre son avenir.

Nous avons dit ci-dessus la vitalité des études de Géographie Régionale, et leur caractère éminemment français. C'est ce que présente R. Musset dans une contribu-

${ }^{38}$ G. Cнавот. La géographie urbaine. (1), pp. 143-147.

${ }^{39}$ voir surtout l'œuvre magistrale de M. SORRE (45), notamment le tome III consacré à l'habitat, véritable manuel de géographie urbaine.

${ }^{40} \mathrm{La}$ géographie agricole, - le miracle annuel de la récolte toujours renouvelée... c'est là l'objet de la géographie agricole - se distance nettement de la géographie agraire qui se préoccupe des problèmes techniques et économiques posés par l'activité agricole, et de l'aménagement de l'espace rural. Cette dernière est fort bien définie par E. JullLaRd, La géographie agraire. (1) pp. 159-166, tandis que la géographie agricole est représentée par le petit livre de P. GEORGE (25) qui a une valeur d'initiation.

${ }^{41}$ A. GiberT. Géographie de l'industrie. (1), pp. 167-174.

42 voir par exemple (1), p. 167.

${ }^{43}$ L. Champier. Géographie politique et géographie religieuse. (1), pp. 149-158.

${ }^{44}$ R. Dion. La géographie historique. (1), pp. 183-186.

45 (1), pp. 187-196. 
tion ${ }^{45}$ qui s'attache d'abord à mettre en valeur les difficultés que rencontrent la création et la parution de monographies régionales: diversité et complexité des problèmes, définition et limite de la notion de région, conditions matérielles de publication. De ces difficultés résulte un certain renoncement à l'élaboration d'études régionales complètes ${ }^{46}$ au profit de sujets régionaux particuliers, notamment sur la morphologie en géographie physique, sur l'organisation rurale et la vie agraire en géographie humaine.

$\mathrm{Si}$, dignes successeurs des fondateurs de la géographie française les géographes français du milieu du $X X^{\mathrm{e}}$ siècle continuent à se pencher avec quel intérêt sur le sol natal, leurs travaux et leurs recherches hors des frontières nationales se sont particulièrement développés ces dix dernières années. C'est incontestablement la première remarque qui s'impose à la lecture des dix contributions consacrées aux diverses régions du globe terrestre.

Les géographes français ont en général pris moins d'intérêt à l'Europe ${ }^{47}$. Les études d'ensemble sont rares et chaque région européenne a ses géographes. Quand on parle des Alpes, par exemple, c'est surtout à l'Ecole de Grenoble et à R. BLANCHARD (9) qu'il faut se référer. Si l'Allemagne a connu peu de faveurs, il en va tout autrement de la Péninsule ibérique qui a attiré et attire toujours un grand nombre de chercheurs, de même que l'Europe méditerranéenne.

Avant la deuxième guerre mondiale, la géographie de la Russie était surtout connue dans les pays de langue française par les travaux de L. RAveneau et de P. CAmena D'Almeida. Depuis 1945, grâce à P. George notamment, l'Ecole française a accompli un effort particulier pour faire connaitre en Occident aussi bien l'URSS elle-même que l'abondante production soviétique, sans oublier les Républiques populaires ${ }^{48}$.

Mais, c'est dans les Territoires de l'Union française que l'activité des géographes français s'est multipliée. Ainsi, l'Afrique du Nord ${ }^{49}$ semble mieux connue que beaucoup d'autres régions du globe. En effet, ouvrages généraux $(21,13)$ et études régionales sont aussi précieux que nombreux, tandis que les publications spécialisées, tant en géographie physique qu'en géographie humaine, abondent. L'étude des mouvements récents de l'écorce terrestre et des changements de climats mobilise le plus grand nombre de chercheurs tandis que du côté de la géographie humaine, les monographies régionales sont plutôt œuvres d'historiens, de sociologues ou d'ethnographes. Toutefois, ces dernières années, colonisation agricole, pêche, colonisation minière, géographie économique en général et surtout géographie sociale et géographie de la population (15) attirent de plus en plus les géographes.

L'étendue des territoires relevant de l'Union française ou de la France, le nombre, la complexité et l'évolution des problèmes de ces immenses régions n'ont pas empêché les géographes français de s'intéresser aux autres territoires africains ${ }^{50}$. Cet intérêt relève surtout du besoin d'établir des comparaisons élargissant et permettant l'établissement de conclusions plus valables: géographie humaine du Fezzan, précision sur l'Egypte (problème de l'eau), croissance des villes d'Afrique occidentale, recherches sur le peuplement de l'Afrique, le problème démographique et racial en Afrique du Sud, etc., etc.

Le Moyen-Orient ${ }^{51}$, l'Asie des Moussons et l'Asie Centrale ${ }^{52}$ ont toujours inté-

${ }^{46}$ Des thèses et des ouvrages importants de type ancien continuent cependant à paraitre: (23), (9), (36).

47 J. Sermet. Les géographes français et l'Europe. (1), pp. 197-210.

48 P. George. Etudes françaises sur la géographie de l'U.R.S.S. (1), pp. 211-215. - Etudes françaises sur les Républiques populaires européennes. (1), pp. 217-218.

49 J. Dresch. Etudes sur l'Afrique du Nord. (1), pp. 219-229.

50 M. Larnaude. Travaux géographiques publiés par des Français sur l'Afrique non française (1945-1955). (1), pp. 231-235.

51 E. DE Vaumas. Le Moyen-Orient. (1), pp. 237-244.

52 C. Robequain. Asie des Moussons et Asie Centrale. (1), pp. 257-260. 
ressé de manière particulière les Français, comme le prouve l'importante bibliographie que publient E. DE VAumas et C. Robequain. Par contre, les études américaines ${ }^{53}$ ont connu un sort différent. Parmi les ouvrages d'ensemble, il faut signaler le livre de J. Gottmann (29). Les travaux sur le continent nord-américain sont moins nombreux qu'avant-guerre. Notons toutefois, à côté de plusieurs études d'hydrologie fluviale, de géographie de la population et de géographie économique sur les Etats-Unis comme sur le Canada, «une manière de chef d'œuvre» le «Tableau des Etats-Unis» brossé par A. Siegfried (43).

L'intérêt pour l'Amérique latine date d'un peu plus d'une vingtaine d'années, et c'est surtout au Brésil que les géographes français ont enseigné et travaillé. Parler du Brésil, c'est obligatoirement mettre en valeur un des caractères les plus frappants de l'Ecole française de géographie: son caractère formateur. Non seulement de très nombreux géographes étrangers ont appris leur métier en France, mais encore, de tout temps, des dizaines de professeurs français enseignent et dirigent des disciplines et des instituts géographiques dans d'autres pays ${ }^{54}$. E. DE Martonne, entre autres, entraîna en 1937 les jeunes géographes brésiliens à la demande de l'Université de Sao Paulo. P. Deffontaines dirige depuis 1940 l'Institut français de Barcelone.

En plein développement sur les vieux continents, la recherche géographique française se devait également d'aborder les régions polaires ${ }^{55}$. C'est chose faite sur une vaste échelle depuis 1947 et grâce à P.-E. VICToR. Certes, la tradition polaire en France est antérieure ${ }^{56}$ et plus d'un ouvrage $(10,38)$ toujours précieux, traitant de géographie physique et d'ethnographie, a paru avant le départ des Expéditions Polaires Françaises qui furent lancées avec le concours des pouvoirs publics et pour des motifs strictement scientifiques dès 1948. Ces expéditions, grâce à leurs effectifs (parfois 30 à 40 hommes), aux moyens techniques modernes (chenillettes, support aérien) et surtout, à la compétence et au courage de leurs membres, ont obtenu des résultats très importants qui représentent une contribution que l'on peut qualifier de fondamentale.

Ces résultats ${ }^{57}$ touchent les travaux cartographiques et géodésiques qui ont permis de dresser une première carte altimétrique de la partie centrale et méridionale de l'inlandsis au 1:5000 000, d'en fixer le point culminant à environ 3220 mètres sur mer et à une centaine de kilomètres au Nord-Est de la Station centrale ${ }^{58}$. Les études séismiques ont mis en évidence le relief et l'hypsométrie du socle sous-glaciaire, tandis que les opérations gravimétriques assurèrent, entre autres, le recoupement des résultats fournis par les données séismiques. Les mesures glaciologiques ont porté sur les phénomènes d'accumulation et d'ablation - le bilan total de l'inlandsis est négatif de cent kilomètres cubes - sur la dynamique du glacier - vitesse d'écoulement journalier de glaciers actifs grœnlandais: trois à dix mètres - sur l'altitude moyenne de la ligne du névé - environ $1600 \mathrm{~m}$. s./m. au Grœenland - sur la masse de la glace inlandsisienne qui est, selon $A$. BAUER, de $2,6 \times 10^{6}$ kilomètres cubes, ce qui revient à dire que toute la fonte de cette masse de glace équivaudrait, toujours selon BAUER, à une tranche d'eau de 7 mètres répartie sur l'ensemble des mers du globe. Le dépouillement des études météorologiques permet d'ores et déjà les premières conclusions suivantes:

53 R. Blanchard. Amérique anglo-saxonne. (1), pp. 245-247.

P. Monbeig. Les géographes français et l'Amérique latine. (1), pp. 249-256.

${ }^{54} \mathrm{La}$ Suisse romande ne saurait oublier les noms de J. Brunhes (1869-1930) et de P. GirarDIN (1875-1950) professeurs de géographie et ancien recteur de l'Université de Fribourg.

${ }^{55} \mathrm{~J}$. Malaurie. L’activité géographique française dans les régions polaires $(1940-1955)$. (1), pp. $261-280$.

${ }^{56}$ Les expéditions du Dr J. Charcot datent de 1903 et 1908 , et la Bibliographie des travaux français relatifs à l'année polaire 1932-1933 est des plus abondantes.

${ }^{57}$ L'ensemble des publications définitives des Expéditions Polaires Françaises comprendra trois séries : série N, Expéditions Arctiques, série S, Expéditions Antarctiques et série G, Sujets de Technique générale, séries à leur tour subdivisées en 6 ou 8 groupes.

58 Sa position géographique est: $70^{\circ} 54^{\prime} 50^{\prime \prime} \mathrm{N}-40^{\circ} 38^{\prime} 00^{\prime \prime} \mathrm{W}$. 
a) extrêmes enregistrées (1949-1950) - $64^{\circ} \mathrm{S} \mathrm{C}$. (minimum) et $+00^{\circ} 7 \mathrm{C}$. (maximum); b) le temps peut être instable et connaitre de forts écarts de température: $29^{\circ}$ C. en un jour, $14^{\circ}$ C. en trois heures; c) les précipitations ont atteint en dix mois (1950-1951) à la Station centrale: $70 \mathrm{~cm}$. de hauteur de neige, soit $130 \mathrm{~mm}$. d'eau pour cinq mois et demi; d) les températures moyennes annuelles enfin ont été de même ordre en 1930-1931 et 1949-1951.

Les études de géographie physique sur la côte occidentale du Græenland ont touché la climatologie, la biogéographie, la morphologie, tandis que la Mission géographique française à Thulé, 1950-1951, conduite par J. MALAURIE, s'attachait surtout à la démographie de cette région.

La France ne possède aucun territoire dans l'Arctique. Par contre, dans l'hémisphère sud, grâce à la découverte de D. D’URville en 1840, elle dispose de la TerreAdélie. D'accès difficile, de climat parmi les plus pénibles de l'Antarctique, le pays était presque encore à découvrir au débarquement des Expéditions Polaires Françaises en 1949. Six ans plus tard, nous disposons d'études et de documents 59 aussi divers que précieux tant en cartographie, géologie, océanographie, physique du globe, glaciologie, météorologie que biogéographie, études que seule la technique moderne pouvait permettre de réaliser.

Pour compléter le grandiose panorama de La Géographie française au milieu du XX" siècle, «l'active équipe dirigeante de la revue l'Information géographique» se devait de signaler ceux qui soutiennent, favorisent et permettent la recherche d'une part, les documents, les collections et les institutions qui diffusent, rappellent et conservent ces recherches et leurs résultats d'autre part.

Fondé en 1939, le Centre National de la Recherche Scientifique 60 compte parmi ses trente et une sections ou disciplines, une section de géographie. Si le nombre des chercheurs géographes est relativement peu élevé (28 sur un effectif d'environ 2500 chercheurs en décembre 1953), c'est qu'un effort tout particulier a été fait au titre des frais de missions et de séjour en France et à l'étranger 61 . De grands progrès sont réalisés en même temps dans l'équipement de nombreux laboratoires de géographie, notamment dans les domaines de la cartographie et de la photographie. Le C. N. R. S. participe encore aux frais de publication de revues, d'ouvrages et de thèses. Il organise des colloques de spécialistes sur des problèmes précis et actuels. Il a créé enfin le Centre de documentation cartographique et géographique auprès de l'Institut de géographie de Paris. Ainsi, le C. N. R. S. joue un rôle considérable dans la recherche géographique française, car, aujourd'hui, «aucune publication d'envergure, aucune mission lointaine, aucun équipement important ne peut être laissé à la charge des individus».

Nous venons de voir que le C. N.R.S. facilite de plus en plus la conduite matérielle de la recherche. Ajoutons immédiatement que la diffusion de cette dernière est assurée en grande partie par quelques dizaines de revues 62 qui, par leur diversité, la qualité des travaux qu'elles publient, l'originalité des préoccupations dont elles témoignent n'ont peut-être nulle part leur équivalent.

Jusqu'à la fin du $\mathrm{XIX}^{\mathrm{e}}$ siècle, les publications géographiques régulières n'étaient guère représentées que par les bulletins des sociétés de géographie 63. Signalons la principale: La Géographie (1875-19o1), revue de la Société de Géographie de Paris. Or, dix ans auparavant, P. VIDAL DE LA BLAChe fondait les Annales de Géographie dont la LXV Année (1956) est en cours de parution, représentant quelque trois cent-cinquante fascicules (5). Dirigées par un collège de maîtres de l'Ecole française, les Annales demeurent fidèles à la géographie régionale, donnent d'importants articles de géographie générale et de méthodologie, s'efforcent, par des notes et des comptes rendus, de suivre le mouvement géographique français et étranger.

Cependant, les Annales ne pouvaient suffire seules à la tâche. Aussi, au fur et à mesure de la création de chaires de géographie dans les Universités et du «succès du nouvel enseignement», de nouveaux moyens d'expression plus décentralisés, devinrent nécessaires. C'est ainsi que naquit le Recueil des travaux de l'Institut de géographie alpine en 1913, devenu sept ans

59 voir note 57.

60 A. Journaux. Le Centre National de la Recherche Scientifique (C.N.R.S.) et la recherche géographique en France. (1), pp. 295-298.

${ }^{61}$ Il a été organisé en 1954: 23 missions en France et 18 à l'étranger.

62 D. Faucher. L'activité géographique française: Les Revues. (1), pp. 299-304.

${ }^{63} \mathrm{Par}$ exemple: Bulletin de la Société de géographie de Lille, Bulletin de la Société de géographie de Marseille, ou le Bulletin de la Société languedocienne de géographie (Montpellier). 
plus tard, sous la direction de R. BLANChARD, la Revue de géographie alpine, aujourd hui, «une des grandes revues internationales de géographie».

Organe de l'Institut du même nom, créé en 1923, les Etudes rhodaniennes, après fusion avec le Bulletin de la Société de Géographie de Lyon en 19+2, sont devenues la Revue de géographie de Lyon sous la direction d'A. Allix. En 193o, apparaît la Revue géographique des Pyrénées et du Sud-Ouest, tandis qu'il y a trois ans était fondée Norois, Revue géographique de l'Ouest et de l'Atlantique Nord.

Ainsi, la première moitié du $\mathrm{XX}^{\mathrm{e}}$ siècle a vu l'éclosion et l'épanouissement des revucs régionales françaises. De plus, il faut signaler, depuis une vingtaine d'années, les efforts faits pour assurer la parution de revues plus spécialisées. La Revue de géomorphologie dynamique - dès 1950 - en est une des illustrations les plus vivantes tandis que L'Information géographique - dès 1936 - s'affirme particulièrement précieuse pour l'enseignement à tous les degrés. Enfin, il faudrait encore pouvoir signaler les très nombreuses publications directement utiles au géographe, comme par exemple, la revue Population, organe de l'Institut national d'études démographiques, institut et revue dirigés par A. SAUvY, ou les Bulletins, Cahiers et Etudes des territoires d'outre-mer, sans oublier les périodiques de bonne vulganisation comme le Larousse mensuel, Geographia, Transmondia, etc.

«La représentation cartographique est partie intégrante du travail géographique». Cette affirmation d'A. Cholley rappellera, à ceux qui en douteraient encore, toute l'importance de la cartographic 64 dans notre discipline. Jusqu'en 194o, la cartographie officielle française était concentrée entre les mains du Service géographique de l'Armée. Devenu organisme civil sous le nom d'Institut géographique national, il crée non seulement le matériel cartographique dont la France a besoin, mais forme encore lui-même un personnel scientifique et technique hautement qualifié. Comme en Suisse, la cartographie privée joue un rôle de premier plan65, notamment dans la publication d'Atlas généraux dont certains sont devenus classiques $(6,7)$.

Livres, périodiques, cartes et photographies forment la documentation de base de tout chercheur et du géographe en particulier. Or, les bibliothèques françaises 66 sont aussi nombreuses que riches, notamment dans le domaine géographique. Fondée en 1821, "La bibliothèque de la Société de géographie 67 est, sans conteste, la plus importante bibliothèque géographique française et une des plus riches du monde». Chaque mois, elle publie une bibliographie 68. Tous les instituts, la plupart des ministères, les grandes institutions économiques et culturelles possèdent des fonds plus ou moins importants de documentation géographique, de plus en plus souvent subdivisés en bibliothèques, cartothèques et photothèques 69 .

L'extraordinaire développement de la documentation pose, parmi bien d'autres, le problème du regroupement de cette documentation en vue de la consultation. De nombreux efforts 67 ont été opérés dans ce sens pour le plus grand bien des chercheurs certes, mais aussi, dans l'intérêt même de la recherche.

La Géographie française au milieu du $X X^{e}$ siècle ne pouvait s'achever sans faire allusion à ces deux œuvres d'un intérêt international: la Bibliographic géographique internationale 70 et la Bibliographie cartographique internationalc 71 , dont la première en tout cas est œuvre essentiellement française.

Créée en 1890 sous le nom de Bibliographie géographique annuelle, elle n'est devenue internationale que depuis le Congrès international de géographie tenu à Paris en 1931. La fermeture des frontières, le manque de collaborateurs et de liaisons parfois, les difficultés financières faillirent compromettre cette œuvre indispensable. Le volume 1951-1953 a paru en 1956, et l'on peut espérer le volume 1954-1955 pour le début de cette année. Souhaitons que des collaborateurs de plus en plus nombreux assurent un travail bibliographique de plus en plus large et de plus en plus valable du seul point de vue scientifique.

${ }^{64}$ A. Libault. Vingt années de cartographie française. (1), pp. 305-313.

${ }^{65}$ Chacun a pu apprécier un jour ou l'autre toutes les qualités de la célèbre carte routière Michelin au 1:200000, certainement un des exemples les plus connus et les meilleurs de la production privée de la cartographie française.

${ }^{66}$ M. Foncin. La documentation géographique dans les bibliothèques françaises. (1), pp. 315 - 321 .

${ }^{67}$ "Installée depuis 1942 à la Bibliothèque nationale dans le même bâtiment que le département des Cartes et Plans dont elle partage la salle de travail (58, rue Richelieu, Paris 2e), elle compte environ 400000 volumes et 500 périodiques en cours, une très belle collection d'atlas, des cartes, des manuscrits et des portraits d'explorateurs."

68 Pour la recherche bibliographique, voir aussi (39).

69 "Le Centre de documentation de photographie aérienne ouvert en 1945 à l'Institut géographique national centralise tous les clichés aériens pris par ou pour les services publics en France et dans l'Union française. Actuellement plus de 700000 clichés."

${ }^{70}$ P. George. La Bibliographie géographique internationale. (1), pp. 323-325.

“ M. Foncin et P. R. Sommer. La Bibliographie cartographique internationale. (1), pp. 327-329. 
C'est en 1938 que l'Union géographique internationale décida «qu'une Bibliographie cartographique internationale serait publiée sous les auspices de l'U. G. I. suivant les principes adoptés pour la Bibliographie cartographique de la France présentée au Congrès par le Comité national français de géographie». Le premier volume (1946-1947) n'a paru qu'en 1949 et présente les cartes publiées par huit Etats et leurs territoires d'outre-mer. On dispose actuellement de sept volumes, dont le dernier (1954) présente des documents de vingt et un pays: Allemagne, Argentine, Autriche, Belgique, Brésil, Canada, Danemark, Etats-Unis, Finlande, France, Grande-Bretagne, Israël, Italie, Japon, Mexique, Norvège, Pays-Bas, Portugal, Suède, Suisse et Turquie.

Chose bizarre, ce sont surtout des difficultés de collaboration et d'organisation et non point d'ordre matériel et financier qui ont entravé l'œuvre jusqu'à aujourd'hui. Ceci est de bon augure pour l'avenir de la Bibliographie cartographique internationale 72.

Que conclure au terme de ces quelques pages bien trop sommaires et incomplètes face à ce premier demi-siècle de magnifique épanouissement de l'Ecole française de géographie? Qu'en sera-t-il du second?

Il est aisé de répondre à notre première question! On ne peut qu'être émerveillé en face de tant d'honnête et puissant labeur, reconnaissant à tous ceux qui ont consacré le meilleur d'eux-mêmes «à une description raisonnée», c'est-à-dire une tentative d'explication de la Terre et de l'Homme, connaissance sans laquelle il ne sera point de paix durable ${ }^{73}$, tant il est vrai que l'ignorance est un des pires ennemis de l'humanité; on ne peut qu'espérer voir la Géographie occuper de plus cn plus la place qui lui revient dans le concert des sciences, rejetant à jamais la triste affirmation de L. Vivien de Saint-Martin, il y a trois quarts de siècle.

Mais, cette place ne sera pleinement occupée que si la Géographie reste fidèle à elle-même, une, tout à la fois régionale et générale, physique et humaine, véritable lien entre les spécialistes des diverses spécialités qui poursuivent, chacun dans son domaine et selon sa méthode et ses techniques, ce but commun d'une meilleure connaissance de la Terre et de l'Homme. Cette tendance, nouvelle certes en France, mais déjà bien marquée est le fait d'une géographie appliquée ${ }^{74}$, utilitaire, qui doit peu à peu faire et gagner sa place dans le faisceau des disciplines qui forment la Géographie moderne, et ceci, dans l'intérêt même de la recherche pure qui prendra par là une nouvelle et plus grande importance.

C'est dans cette direction que l'Ecole française de géographie, formée, limitée et sans cesse repensée au cours de ces cinquante dernières années, semble devoir s'affirmer dans la seconde moitié du $X^{\mathrm{e}}$ siècle.

72 L'Uruguay et la Yougoslavie seront sans doute représentés dans le tome VIII (1955).

73 "Je persiste à croire qu'au jugement politique seraient bien nécessaires les informations fondamentales qu'est en mesures d'apporter le géographe." M. LE LANnou. 1 art. in "Le Monde", 10 novembre 1956.

${ }^{74}$ A. Meynier. La géographie appliquée. (1) pp. $281-287$.

\section{BIBLIOGRAPHIE SOMMAIRE}

(1) Information Géographique (L'). La Géographie Française au milieu du XXe siècle. J.-B. Baillière \& fils, Paris, 1957. 1 vol. 333 p. - (2) Allix A. et GiberT A.: Géographie des Textiles. Coll. de Géographie économique et sociale. Librairie de Médicis, Paris 1957.1 vol. 572 p. - (3) ANcEL, J.: Géographie des Frontières. Coll. de Géographie humaine, vol. $\mathrm{N}^{\circ} 12$. Gallimard, Paris, 1938. 1 vol. avec pl., 210 p. - (4) Ancel, J.: Manuel géographique de politique européenne. Delagrave, Paris, 1937-1945. 3 vol., ill., cartes. - (5) Annales de Géographie: Bulletin de la Société de Géographie (de Paris). Revue paraissant 6 fois par an. Directeurs: A. Cholley, M. Sorre, J. Dresch. 4 Tables décennales (analytiques), 1891-1931. Dès le 15 octobre 1891. A. Colin, Paris. - (6) Atlas historique et géographique Vidal-La Blache: A. Colin, Paris. 1 vol. de 385 cartes et cartons; avec 1 index de 32000 noms. - (7) Atlas classique de Géographie ancienne et moderne: Publié sous la direction de F. Schrader et L. Gallouedec. Hachette, Paris. 1 vol. de 343 cartes; avec figures, graphiques et index. - (8) Beaujeu-Garnier, Jacqueline: La Géographie de la Population. Coll. de Géographie économique et sociale. Paris 1956, 1 vol., 436 p. - (9) Blanchard, R.: Les Alpes occidentales. P. Arthaud, Grenoble, 1938-1954. 11 vol. - (10) Bruet, E.: L'Alaska. Payot, Paris, 1945. 1 vol. avec 
figures, cartes, statistiques et bibliographie, 451 p. - (11a) BRUNHEs, J.: La Géographie humaine. Félix Alcan, Paris (1ère édit. 1910), 1925. 3 vol. avec fig., pl., cartes. (11b) BRUNHes, J.: La Géographie humaine. Edition abrégée mise au point par $\mathrm{M}^{\text {me }} \mathrm{M}$. Jean-Brunhes, Delamarre et Pierre Deffontaines. Presses Universitaires de France, Paris, 1942, 1 vol. avec cartes, pl., index et bibliographie, 365 p. - (12) CAPOT-Rey, R.: Géographie de la circulation sur les continents. Coll. de Géographie humaine, vol. $N^{\circ}$ 20. Gallimard, Paris, 1946. 1 vol. avec pl. - (13) CAPOT-Rer, R.: Le Sahara français. Coll. Pays d'outre-mer. Presses Universitaires de France, Paris 1953. 1 vol. avec fig., pl. et cartes, 568 p. - (14) Cнавот, G.: Les Villes. Aperçu de géographie humaine. Coll. Armand Colin, vol. $\mathrm{N}^{\circ}$ 250. A. Colin, Paris, 1948. 1 vol. - (15) Chevalier, L.: Le problème démographique nord-africain. Coll. Travaux et documents, Cahier $\mathrm{N}^{\circ} 6$ de l'Inst. national d'ét. démographiques. Presses Universitaires de France, Paris, 1947. 1 vol. avec 5 fig. 221 p. - (16) CHOLI.EY, A.: Emmanuel de Martonne. 1 art. nécrologique dans Annales de Géographie, $\mathrm{N}^{\circ} 347$ (JanvierFévrier 1956), pp. 1-14. Avec 1 portrait h.t. - (17) Cholley, A.: Guide de l'étudiant en géographie. Presses Universitaires de France, Paris 1942. 1 vol. avec bibliographie. 231 p. - (18) CLERGET, P.: Les Industries de la Soie en France. Coll. Armand Colin, vol. $\mathrm{N}^{\circ}$ 61. A. Colin, Paris, 1925. 1 vol. avec graphiques et tableaux statistiques. - (19) Deffontaines, P.: Géographie et Religions. Coll. de Géographie humaine, vol. $\mathrm{N}^{\circ}$ 21. Gallimard, Paris, 1948. 1 vol. avec pl. et cartes, 439 p. - (20) Demangeon, A.: Problèmes de géographie humaine. A. Colin, Paris, 1942. 1 vol. avec 40 fig. Avec 1 biographie et 1 bibliographie d'Albert Demangeon par E. de Martonne. 407 p. - (21) Despors, J.: L'Afrique du Nord. Coll. Colonies et Empires, 4e série : Géographie de l'Union française. Presses Universitaires de France, Paris, 1949. 1 vol. avec fig., pl., cartes et 1 bibliographie. 624 p. - (22) Dıos, R.: Les Frontières de la France. Hachette, Paris, 1947. 1 vol. (23) Fenelon, P.: Le Périgord. Etude morphologique. Thèse de l'Université de Paris. 1952. 1 vol. avec fig., pl. et cartes. 527 p. - (24) GEorge, P.: La campagne. Le fait rural à travers le monde. Presses Universitaires de France, Paris, 1956. 1 vol. avec 29 cartes et fig. et 8 pl. h.t., 397 p. (25) George, P.: Géographie agricole du monde. Coll. Que sais-je ? vol. $\mathrm{N}^{\circ} 212$. Presses Universitaires de France, Paris, 1946. 1 vol. avec 6 fig. 128 p. - (26) GEorge, P.: Géographie de l'Energie. Coll. de Géographie économique et sociale. Librairie de Médicis, Paris, 1950. 1 vol. avec fig., pl., index et bibliographie. 469 p. - (27) George, P.: Introduction à l'étude géographique de la population du monde. Coll. Travaux et documents, Cahier $\mathrm{N}^{\circ} 14$, de l'Inst. national d'ét. démographiques. Presses Universitaires de France, Paris, 1951. 1 vol. avec fig. et 1 bibliographie. (28) George, P.: La Ville. Le fait urbain à travers le monde. Presses Universitaires de France, Paris, 1952. 1 vol. avec fig., pl., cartes et tableaux. 400 p. - (29) Gottmans, J.: L'Amérique. Coll. Les cinq parties du Monde. Hachette, Paris, 1949. 1 vol. avec cartes, fig. et 1 bibliographie. 451 p. - (30) Gottmann, J.: La politique des Etats et leur géographie. A. Colin, Paris, 1952. 1 vol. 228 p. - (31) Guil.cher, A.: Morphologie littorale et sous-marine. Coll. Orbis, Introduction aux études de géographie. Presses Universitaires de France, Paris, 1954. 1 vol. avec 40 figures et 8 ph. h. t., avec des orientations bibliographiques. 216 p. -- (32) LAPPARENT, A. DE: Leçons de géographie physique. Masson, Paris, 1907. 1 vol. avec fig., cartes et lexique des noms et des matières. 728 p. - (33) Lauga, H.: De la banquise à la jungle. Les Français, la terre et les hommes. Textes et documents réunis par... Plon, Paris, 1952. 1 vol. avec 1 carte et 1 croquis. $250 \mathrm{p}$. (34) Lavedan, P.: Géographie des Villes. Coll. de Géographie humaine, vol. N ${ }^{\circ}$ 9. Gallimard, Paris, 1936. 1 vol. avec pl., fig. et cartes. 206 p. - (35) Lavedan, P.: Histoire de l'urbanisme. H. Laurens, Paris, 1926-1952. 3 vol. avec fig., pl., plans, cartes et diagrammes. - (36) Le Lannou, M.: Géographie de la Bretagne. Plihon, Rennes, 1950-1952. 2 vol. pl. et cartes. - (37) Le Lannou, M.: La Géographie humaine. Coll. Bibliothèque de Philosophie Scientifique. Flammarion, Paris, 1949. 1 vol. avec 1 bibliographie. 252 p. - (38) Lerol-Gourhan, A.: Archéologie du Pacifique-Nord. Paris, 1946. 1 vol. avec fig. et cartes. 542 p. - (39) MarTonNe, E. DE: Manuel de la recherche documentaire en France, tome II : Géographie. Publié sous la direction d'... Presses Universitaires de France, Paris, 1946. 1 vol. avec des index d'Auteurs, de Titres, de Sujets, 3 cartes h.t. 103 p. (40) Martonne, E. DE: Traité de géographie physique. A. Colin, Paris 1ère édit. 1909, 9c édit. 1951. 3 vol. avec fig., pl., index. - (41) Schrader, F. et Gallouedec, L.: Atlas classique, voir (7). (42) Siegrried, A.: Géographie électorale de l'Ardèche sous la Troisième République. Coll. Cahiers de la Fondation nationale des sciences politiques, vol. 9. A. Colin, Paris, 1949. 1 vol. avec cartes, diagrammes et tableaux. 140 p. - (43) Siegfried, A.: Tableau des Etats-Unis. A. Colin, Paris, 1954. 1 vol. cartes, diagr. 348 p. - (44) SiegifrifD, A.: Tableau politique de la France de l'Ouest sous la Troisième République. Paris, 1913. 1 vol. avec cartes. - (45) Sorre, M.: Les fondements de la géographie humaine. A. Colin, Paris, 1943-1952. 4 vol. avec fig. et index alphabétique et analytique des matières. - (46) SORRE, M.: Les migrations des peuples. Essai sur la mobilité géographique. Coll. Bibliothèque de Philolophie Scientifique. Flammarion, Paris, 1955. 1 vol. avec notes bibliogr. 265 p. - (47) VIDAL DE LA BLACHE, P.: Atlas historique et géographique VidalLa Blache, voir (6). - (48) VIDAL DE LA BLACHE, P.: Principes de géographie humaine. Publiés d'après les manuscrits de l'auteur par Emmanuel de Martonne. A. Colin, Paris, 1921. 1 vol. 329 p. (49) Vivien DE SAINT-MarTin, L.: Histoire de la géographie et des découvertes géographiques depuis les temps les plus reculés jusqu'à nos jours. Hachette, Paris, 1873. 1 vol. avec 1 atlas et 2 index analytique et alphabétique. $615 \mathrm{p}$. 
Der Verfasser entwirft auf der Basis des vor kurzem erschienenen Rechenschaftsberichtes «La Géographie Française au milieu du XX $X^{\mathbf{e}}$ siècle» (Paris 1957) einen Rück- und Ausblick auf das moderne geographische Schaffen in Frankreich. Die Bilanz zeigt, daß die französischen Geographen auf so gut wie allen Gebieten ihres Faches Bemerkenswertes geleistet haben, in der allgemeinen Geographie wie in der Länderkunde, in den analytischen Bereichen wie in der Synthese, über heimatliche Themen wie über das europäische und außereuropäische Ausland. Obwohl bewußt die «Einheit»der Disziplin im Zentrum stand, war keineswegs Uniformität der einzelnen Arbeiten, sondern optimale Vielfalt die Folge. Dabei ist zweifellos - wie übrigens in andern Ländern — die Tendenz zu verstärkter Pflege der Anthropogeographie im weitesten Sinne (Wirtschaftsgeographie, Politische Geographie, Religionsgeographie, Sozialgeographie, Industriegeographie, Stadtgeographie usw.) zu erkennen, die sich auch im regionalen Sektor deutlich ausprägt. $\mathrm{Da} B$ in der Tat von einer Progression gesprochen werden kann, verdeutlichen in der Berichtszeit neu entstandene Institutionen wie das Nationalzentrum für wissenschaftliche Forschung, das Zentrum für kartographische und geographische Dokumentation sowie diverse Zeitschriften, die auch die starke Spezialisation erkennen lassen, die ähnlich wie andere Disziplinen neuerdings die Geographie durchdringt.

\section{ZUR SYSTEMATIK DES TROPENKLIMAS}

\section{Paul Schaufelberger}

\section{EINLEITUNG}

Es mag weite Kreise überraschen, daß man um die Mitte des 20. Jahrhunderts hinsichtlich der Beurteilung des Tropenklimas noch zu keiner befriedigenden einheitlichen Lösung gelangt ist. Doch stößt man trotz der bekannten Klimatatsachen auf folgende teils sich widersprechende Ansichten.

A. Hinsichtlich der Klimafaktoren und -elemente zeigt sich:

1. Grenzen der Tropen.

a) Mathematisch werden die Tropen durch die Wendekrcise begrenzt.

b) Zweifellos liegen in der Nähe dieser Parallelkreise bestimmte Jahresisothermen, wie diejenigen von 18 oder $20^{\circ}$, die ebenfalls die Grenze der Tropen erkennen lassen.

2. Temperaturen.

a) Infolge der Begrenzung der Tropen durch bestimmte Jahresisothermen hat man sich verführen lassen, die Tropen als heisse Klimazone zu definieren.

b) Bekannt ist aber auch die Tatsache der Gleichförmigkeit der mittleren Monatstemperaturcn, ganz unabhängig von deren absoluten Höhc. Dies trifft für die heisscn und kühlen Tropen zu.

3. Niederschlag.

a) Nach der Regenverteilung werden trockcuc, wechsclfcuchtc und immerfeuchte Tropen unterschieden.

b) Nach der Regenmenge werden die Tropen als arid, scmiarid, semihumid, humid und perhumid beurteilt.

Jeder, der sich mit den Tropen wissenschaftlich befaßt, hat sich daher zu entscheiden, welchen grundsätzlichen Standpunkt or wählen will. Je nach persönlicher Erfahrung oder dem Glauben an gewisse Autoritäten wird er die obigen Klimaelemente so oder so gruppieren. Die Folge sind 2.2. 2. $=8$ verschiedene Hypothesen hinsichtlich der Tropenklimate.

Naturgemäß dürfte nur cine Auffassung die richtige sein, so daß mindestens 7 Arbeitshypothesen sich als revisionsbedürftig erweisen. Kommt nun ein Beobachter, der sich einer dieser revisionsbedürftigen Annahme verschrieben hat, aus der gemäßigten Zone in die Tropen, so wird er andere Verhältnisse antreffen, als er erwartete. Er ist dann enttäuscht und kann sich nicht zurechtfinden. Er kommt leicht zum Schluß, daß die Klimavcrhältnisse in den Tropen anders geartet sind, als wie er sie sich im Vergleich mit der gemäßigten Zone vorstcllte. Er ist deshalb überzeugt, daß zur Erforschung der Tropen spezielle Arbeitsmethoden notwendig sind. Daraus ergibt sich ein weiteres Widerspruchspaar hinsichtlich

B. Hinsichtlich der Arbeitsmethode zeigt sich:

1. In den Tropen gelten dieselben Methoden wie in den übrigen Klimabereichen, d.h. man mu $\beta$ parallelisieren.

2. Die Erforschung der Tropen erfordert spezielle Arbeitsmethoden, d. h. man darf nicht vergleichen oder parallelisieren. 